\title{
Cosmic ray transport and anisotropies to high energies
}

\author{
P. L. Biermann ${ }^{1,2,3,4}$, L. I. Caramete ${ }^{5}$, A. Meli ${ }^{6}$, B. N. Nath ${ }^{7}$, E.-S. Seo ${ }^{8}$, V. de Souza ${ }^{9}$, and \\ J. Becker Tjus ${ }^{10}$ \\ ${ }^{1}$ MPI for Radioastronomy, Bonn, Germany \\ ${ }^{2}$ Department of Physics, Karlsruhe Institute for Technology KIT, Karlsruhe, Germany \\ ${ }^{3}$ Department of Physics \& Astronomy, University of Alabama, Tuscaloosa, AL, USA \\ ${ }^{4}$ Department of Physics \& Astronomy, University Bonn, Bonn, Germany \\ ${ }^{5}$ Institute for Space Sciences, Bucharest, Romania \\ ${ }^{6}$ Department of Physics and Astronomy, University of Gent, Gent, Belgium \\ ${ }^{7}$ Raman Research Institute, Bangalore, India \\ ${ }^{8}$ Department of Physics, University of Maryland, College Park, MD, USA \\ ${ }^{9}$ University de São Paulo, Inst. de Física de São Carlos, São Carlos, Brazil \\ ${ }^{10}$ Department of Physics, University Bochum, Bochum, Germany
}

Correspondence to: P. L. Biermann (plbiermann@mpifr-bonn.mpg.de)

Received: 9 May 2015 - Revised: 14 August 2015 - Accepted: 9 September 2015 - Published: 2 October 2015

\begin{abstract}
A model is introduced, in which the irregularity spectrum of the Galactic magnetic field beyond the dissipation length scale is first a Kolmogorov spectrum $k^{-5 / 3}$ at small scales $\lambda=2 \pi / k$ with $k$ the wave-number, then a saturation spectrum $k^{-1}$, and finally a shock-dominated spectrum $k^{-2}$ mostly in the halo/wind outside the Cosmic Ray disk. In an isotropic approximation such a model is consistent with the Interstellar Medium (ISM) data. With this model we discuss the Galactic Cosmic Ray (GCR) spectrum, as well as the extragalactic Ultra High Energy Cosmic Rays (UHECRs), their chemical abundances and anisotropies. UHECRs may include a proton component from many radio galaxies integrated over vast distances, visible already below $3 \mathrm{EeV}$.
\end{abstract}

\section{Introduction}

The spectrum of Cosmic Rays (CRs) ranges from about a hundred $\mathrm{MeV}$ to a few hundred $\mathrm{EeV}$, with a kink down around a few PeV, the "knee", a second kink up at around $3 \mathrm{EeV}$, the "ankle", and a sharp turn-off around $100 \mathrm{EeV}$ $\left(\mathrm{PeV}=10^{15} \mathrm{eV}, \mathrm{EeV}=10^{18} \mathrm{eV}\right)$. These particles are injected and accelerated in various sites, usually thought to be exploding stars, such as Supernovae (SNe) or Gamma Ray Bursts (GRBs); cf. Abbasi et al. (2012) for energies below a few $\mathrm{EeV}$, and possible activity of super-massive black holes (Active Galactic Nuclei or AGN) at the higher energies, e.g., Gregorini et al. (1984), Biermann and Strittmatter (1987), Witzel et al. (1988), Meli et al. (2008). At their production particles at all energies show a different spectrum, probably flatter, and get steepened by transport through the scattering medium of a turbulent ionized magnetic gas, as the higher energy particles escape faster in what is usually called a "Leaky Box approximation". The spectrum of magnetic irregulari- ties can be directly studied in the Solar wind, where a Kolmogorov law is capable of describing the data (Kolmogorov, 1941a, b; Goldstein et al., 1995). Turbulent energy is injected at some large length scales, producing a saturation spectrum of $k^{-1}$, down to an outer scale $l_{\text {out }}$ of a cascade; from there on down in wavelength (or up in wave-number) the turbulent energy flow in a 3-D-approximation is constant, giving $k^{-5 / 3}$, a Kolmogorov cascade (Kolmogorov, 1941a, b); there may be an intermediate range in wave-numbers between $k^{-1}$ and $k^{-5 / 3}$. This energy flow goes down to the dissipation scale. The data confirm this expectation (Armstrong et al., 1995; Goldstein et al., 1995; Iacobelli et al., 2013; Haverkorn and Spangler, 2013). Does this behavior also hold for the spectrum of magnetic irregularities in the ISM, and how this spectrum extends to the halo, where a wind might be sweeping everything away (Everett et al., 2008, 2012)? Does this have consequences for our interpretation of the sky distribution of the incoming UHECRs (Aab et al., 2015)? 


\section{The irregularity spectrum in the ISM: Kolmogorov?}

The electron density fluctuation spectrum in the ISM matches the expectations from a Kolmogorov description (Armstrong et al., 1995; Haverkorn and Spangler, 2013), with an inner scale between $10^{7}$ and $10^{10} \mathrm{~cm}$, and an outer scale of order $20 \mathrm{pc}$, confirmed by LOFAR (Iacobelli et al., 2013); cf. also Voyager data (Fraternale et al., 2015). CR data allow to test whether a Kolmogorov description is adequate: a Kolmogorov cascade implies that the time-scale for diffusion out of the disk of the Galaxy scales as $E^{-1 / 3}$ : nuclei are observed to run approximately as $E^{-8 / 3}$ (Ahn et al., 2009), electrons approximately as $E^{-10 / 3}$ (Aguilar et al., 2014); electrons are in the loss-limit, i.e. losses due to Synchrotron and Inverse Compton interaction dominate over diffusion (Kardashev, 1962), so their spectrum is expected to be steepened by unity. Therefore the original spectrum for both, nuclei as electrons, is deduced to be $E^{-7 / 3}$, demonstrating consistency, excluding those nuclei, such as $\mathrm{Fe}$, for which spallation losses flatten the spectrum, or that energy range for electrons, where additional processes occur, close to $\mathrm{TeV}$ energies. Error bars in the data do not significantly modify the conclusion. The mean free path $\lambda_{\mathrm{mfp}}$ for scattering of CRs can be written as $\lambda_{\mathrm{mfp}}=\frac{L_{\text {out }}}{b}\left(E /\left\{Z e B L_{\text {out }}\right\}\right)^{1 / 3}$, which is in turn $=10^{17.8} \mathrm{~cm} b_{1 / 3}^{-1} L_{\text {out, } 20 \mathrm{pc}}^{2 / 3}\left(E_{4 \mathrm{GeV}} /\left\{Z B_{5} \mu \mathrm{Gauss}\right\}\right)^{1 / 3}$ where momentum $\mathrm{pc}$ is here approximated as energy $E$ and $Z$ is charge of the $\mathrm{CR}$ nucleus considered, $E_{4 \mathrm{GeV}}$ is that energy in units of $4 \mathrm{GeV}, b_{1 / 3}$ is the fraction of magnetic field energy density in the irregularities in units of $1 / 3, L_{\mathrm{out}} 20 \mathrm{pc}$ is the outer scale of the Kolmogorov range in units of $20 \mathrm{pc}$, and $B_{5} \mu \mathrm{Gauss}$ is the magnetic field strength in units of $5 \mu$ Gauss. The scattering coefficient $\kappa$ can then be written (Drury, 1983) as $\kappa=10^{27.8} \mathrm{~cm}^{2} \mathrm{~s}^{-1} b_{1 / 3}^{-1} L_{\text {out, } 20 \mathrm{pc}}^{2 / 3}\left(E_{4 \mathrm{GeV}} /\left\{Z B_{5 \mu \mathrm{Gauss}}\right\}\right)^{1 / 3}$. This in turn yields an escape time at $4 \mathrm{GeV}$ for a CR disk thickness $H_{\mathrm{CR}, 1.5 \mathrm{kpc}}$ in units of $1.5 \mathrm{kpc}$ of $10^{8.1}$ years $H_{\mathrm{CR}, 1.5 \mathrm{kpc}}^{2} b_{1 / 3}^{+1} L_{\text {out, } 20 \mathrm{pc}}^{-2 / 3} E_{4 \mathrm{GeV}}^{-1 / 3} Z^{+1 / 3} B_{5 \mu \mathrm{Gauss}}^{-1 / 3}$ too long by an order of magnitude (Brunetti and Codino, 2000). One obvious solution is to insert another turbulence regime to add energy at a different wave-number range, between the outer scale of Kolmogorov turbulence, and the very maximum scale, the thickness of the CR disk, or even beyond, and so reduce $b$, the strength of the irregularities just in the Kolmogorov range: this implies that $b$ is then best $b \simeq 0.03$. We note that the lateral radius from where sources contribute is of similar scale as the half width scale height of the CR disk, in the case of isotropic scattering; in case of anisotropic scattering that lateral reach may be larger, reducing any expected anisotropy. Very often, the transport of CRs is deduced from the amount of material it traversed. Since all the nuclei of heavy elements among the
CRs traverse the dense region around the stellar wind and OB-super-bubble (Binns et al., 2008, 2013; Binns, 2011), the interaction may well be decoupled from the transport. Using the CRs themselves as source of excitation for the wavefield in which they scatter, yields an expected grammage $X_{\mathrm{lb}}$-momentum power-law dependence of exponent $-5 / 9$, in addition to the grammage experienced in the average ISM with an exponent of $-1 / 3$ (Biermann, 1998; Biermann et al., 2001, 2009). This is fully consistent with the derivation by Ptuskin et al. (1999): $X_{\mathrm{lb}}=11.3 \mathrm{~g} / \mathrm{cm}^{-2} \beta$ at $R<5 \mathrm{GV}$, and $X_{\mathrm{lb}}=11.3 \mathrm{~g} \mathrm{~cm}^{-2} \beta(R /\{5 \mathrm{GV}\})^{-0.54}$ at $R \geq 5 \mathrm{GV}$, where $\beta c$ is the velocity of the nucleus considered, and $R$ is its rigidity $\mathrm{pc} /\{Z e\}$. There are many caveats with using such a simple isotropic 3-D law as a Kolmogorov $k^{-5 / 3}$ law of turbulence in magnetic field fluctuations as well as in density and velocity fluctuations; (1) magnetic fields are never really isotropic; (2) the ISM has several phases, with vastly different temperatures and densities, with the most tenuous medium of a density of order $10^{-2.5} / \mathrm{cc}$ (Cox and Smith, 1974; Lagage and Cesarsky, 1983; Snowden et al., 1997); (3) transonic flow and shocks may be quite common (Iacobelli et al., 2014). Intermittency may be extreme, stoked by, e.g., stellar explosions, active stars such as micro-quasars (Mirabel, 2010), HII-regions, OBsuper-bubbles, and pulsars; (4) the fraction of magnetic field energy in irregularities differs between spiral arm and inter-arm regions (Beck and Hoernes, 1996); (5) the CR layer thickness may vary strongly throughout a galaxy, small in the inner regions (Biermann et al., 2010), of perhaps only $100 \mathrm{pc}$ or a small multiple thereof, and large outside (Beuermann et al., 1985), of kpc scale; (6) galaxies often have winds (Rossa and Dettmar, 2003; Everett et al., 2008, 2012; Uhlig et al., 2012); (7) such winds are likely to be highly non-stationary, since the stellar activity driving them is often characterized by bursts. These winds are also probably highly unstable to clump formation, like the winds of massive stars, as any driving by a "light fluid", such as photons (massive stars) or CRs (galactic winds) is unstable; (8) driving the irregularity spectrum occurs on many scales. There may be a further outer scale, at which the scattering length becomes independent of energy, or the spectrum runs as $k^{-2}$, possibly driven by shocks. It is amazing, that a simple picture carries us so far (Yan and Lazarian, 2012). Accepting it, we can ask whether the knee in the spectrum of CRs can possibly be caused by a change in propagation: the knee is at an energy of about $2 \mathrm{Z} \mathrm{PeV}$, and its Larmor radius corresponds to a wavelength right in the simple power-law 3-D Kolmogorov spectrum of the irregularity spectrum. So in this approximation the propagation across the knee does not change (e.g. Biermann, 1993; Todero Peixoto et al., 2015), and the knee must be a feature of the sources. 


\subsection{Local CR gradients}

Moving through an isotropic distribution of CRs in their own frame produces an apparent anisotropy, the ComptonGetting effect (Compton and Getting, 1935). The anisotropy is given by $\left\{\delta F_{\mathrm{CR}}\right\} / F_{\mathrm{CR}}=4 \beta \cos \theta$, where $\beta_{\mathrm{c}}$ is the velocity relative to the local system of scattering rest, and $\theta$ is the azimuth relative to the direction of motion. Within the scaleheight of about 1-2 kpc, within the CR-escape time (Brunetti and Codino, 2000) there are about between 1000 and 4000 old Super-Nova Remnants (SNRs) and associated magnetic bubbles, with their shells, and pulsars. Most SNRs are either in cooling, or even later, in the coasting phase. Following Lagage and Cesarsky (1983) and Cox (1972) the final radius of the expanding shell of the cooling phase might reach $300 \mathrm{pc}$ for the density $10^{-2.5} / \mathrm{cc}$, or $30 \mathrm{pc}$ for density unity per cc. So already the injection of CRs may be strongly overlapping (Cox and Smith, 1974), reducing their anisotropy. A corollary question is whether we see in the data all the mottledness of all the nearby CR injection sites (Biermann et al., 2013).

There is another source of information about a possible CR gradient, which would result in an anisotropy: the star formation rate in the Galaxy strongly depends on radial distance from the Galactic Center (GC) (Smith et al., 1978). This star formation and therefore the expected SN-rate strongly peak at the GC. So we expect both the pion contribution of the gamma-ray emission, as well as the local neutrino emission to also show such a peak. The neutrino emission, which statistically cannot be distinguished from isotropy at present (Aartsen et al., 2014), does show a cluster of events near the GC. Assuming the local spectral shape of the CRs, including the knee of the CRs, to also describe the CR spectrum elsewhere in the Galaxy then predicts that the neutrinos ought to have a kink down around $100 \mathrm{TeV}$. This is not seen in the cluster of events near the GC, supporting the doubts about an association of all these events with the GC region.

\subsection{Local Galactic wind?}

A Galactic wind provides a boundary condition for diffusion of GCR particles: Rossa and Dettmar (2003) show that, to cite, "a good correlation exists between the far-infrared flux ratio $\left(S_{60 \mu \mathrm{m}} / S_{100 \mu \mathrm{m}}\right)$ and the Star Formation Rate (SFR) per unit area $\left(L_{\mathrm{FIR}} / D_{25}^{2}\right)$, based on the detections/nondetections". A minimal energy input due to star formation per unit area of $\dot{E}_{25}^{\text {thresh }} \simeq 10^{40.0 \pm 0.3} \mathrm{erg} \mathrm{s}^{-1} \mathrm{kpc}^{-2}$ has been derived, to cite: "Gaseous halos are a direct consequence of Star Formation activity in the underlying galactic disk". Radio observations also demonstrate that many galaxies have winds (Chyźy et al., 2006, 2007). Local energy injection with $N_{\mathrm{SN}, 3}$, the number of local supernovae within about $3 \mathrm{kpc}$ distance within the last 1000 years, is: $N_{\mathrm{SN}, 3}\left(\left\{10^{51} \mathrm{erg}\right\} /\left\{10^{3}\right.\right.$ years $\left.\left.\pi 3^{2} \mathrm{kpc}^{+2}\right\}\right)$ (Tüllmann et al., 2006); this reaches the threshold given if $N_{\mathrm{SN}, 3}>5$, exceeded slightly by known historical supernovae. We inter- pret this as supporting a local Galactic wind (Everett et al., 2008, 2012; Uhlig et al., 2012; Sarkar et al., 2015), with about $1 M_{\odot}$ falling back per year. Beuermann et al. (1985) have derived a model for the CR disk, showing evidence for a thick CR disk, with a half width of $1-2 \mathrm{kpc}$. We interpret the thick disk as the full disk for the confinement of CRs, which provides the inner boundary layer for the wind. Bending in a Parker wind (Parker, 1958) can be an order of magnitude larger than a local estimate would suggest due to integrating the Lorentz force with an $1 / r$ magnetic field. A galactic wind scatters incoming UHECR particles via turbulence with many shocks: an ensuing $k^{-2}$ spectrum in irregularities gives $\kappa_{\text {scatt }}=\operatorname{const}(E)$, so observed UHECR spectra remain unaffected by transport locally.

\subsection{Chemical composition and anisotropy at high energy}

The chemical composition rapidly gets heavier with energy towards a particle energy of order $100 \mathrm{PeV}$ (Gaisser et al., 2013), then gets quickly lighter again, to be compatible with a majority fraction of Hydrogen around 1-3 EeV (Erice CR meeting, 2014): R. Engel, only to rise again to a heavier composition at higher energies (Aab et al., 2014a, b; Todero Peixoto et al., 2015). We can interpret these data using Galactic CRs, a mixed component including heavy elements from the radio galaxy $\mathrm{Cen} \mathrm{A}$, only $50^{\circ}$ from the $\mathrm{GC}$ in direction, and in addition, possibly an extragalactic proton component from radio galaxies all around (Todero Peixoto et al., 2015). The Auger sky suggests a cluster of events around the direction towards Cen A (Kotera and Olinto, 2011), a direction also especially abundant in radio galaxies. So any anisotropy attributable to the GC region and Cen A both ought to show up in the same hemisphere. On the other hand, the TA experiment (Abbasi et al., 2014) detects a hot spot in the sky not very far from the strongest local starburst galaxy M82, suggesting GRBs as a possible source. At energies below $3 \mathrm{EeV}$ and at higher energies we see a directional preference into the opposite direction, at the percent level (Erice CR meeting, 2014): R. Engel; (Letessier-Selvon, 2014): it may correspond to the protons from radio galaxies all around us (Das et al., 2008). If this were true, then with increasing heavy element abundance in the UHECR data (Gaisser et al., 2013), this direction might be distinguishable via the chemical composition. We can interpret the $15^{\circ}$ scattering cloud around the radio galaxy Cen $\mathrm{A}$ in the small angle limit. Using the scale $15 R_{15} \mathrm{kpc}$ as the maximum scale (twice the Sun's Galactic orbit), a charge of $6 Z_{6}$, i.e. Carbon, an energy of $6 \times 10^{19} E_{60 \mathrm{EeV}} \mathrm{eV}$, and $N$ scatterings we find: $N=$ $10^{3.0} R_{15 \mathrm{kpc}}^{2} B_{5 \mu \mathrm{Gauss}}^{2} Z_{6}^{2} E_{60 \mathrm{EeV}}^{-2}$ from the size of this scattering cloud. If we set the scattering length scale again to be of the order of the CR disk scale, we have $N$ of the order 10 to perhaps 100 even in the innermost region of the Galaxy (Biermann et al., 2010); it follows that $B$ is of $\mu$ Gauss level; assuming the cloud of events to be mostly low $Z$ elements implies a stronger magnetic field. The fact that the cloud of 
events is pretty much centered on Cen A also means that the linear bending integrates out to near zero; just the fluctuations produce an angular dispersion. If we accept the chemical composition derived from Auger data (Gopal-Krishna et al., 2010), then other sources further distant are not viable at very high energy. With a model for the wind density and Galactic mass loss (Everett et al., 2012) we can derive further plausible conditions on the wind parameters, and test them. Any widespread proton component may require many sources. Radio galaxies pointed at the observer (Gregorini et al., 1984; Witzel et al., 1988) are recognized as all variable bursting sources (see, e.g, Her A, Gizani and Leahy, 2003): their maximally allowed particle energy can be high (Lovelace, 1976), and so a sufficient number of sources with the required particle energies may exist. Just a few nearby strongly fluctuating bursting sources may dominate the sky (Lemoine et al., 1997); see TA and Auger (Abbasi et al., 2015).

\section{Conclusions}

A model is introduced, in which the irregularity spectrum of the Galactic magnetic field beyond the dissipation length scale is in an isotropic approximation first a Kolmogorov spectrum $k^{-5 / 3}$, then a saturation spectrum $k^{-1}$, and finally a shock-dominated spectrum $k^{-2}$ perhaps mostly in the halo/wind outside the Cosmic Ray disk. Such a model is clearly a simple isotropic approximation. With this model we discuss the Galactic Cosmic Ray spectrum, its anisotropies, as well as the extragalactic ultra high energy Cosmic Rays, their chemical abundances and anisotropies. We have Galactic Cosmic Rays, a component from the radio galaxy Cen A, in direction not far from the Galactic Center, and in addition, possibly an extragalactic proton component from bursting radio galaxies.

Acknowledgements. Todor Stanev has contributed to the evolution of these ideas over very many years. Memberships of some of the authors in the AMS, Auger, H.E.S.S., IceCube, KaskadeGrande, and LOPES collaborations are noted. Discussions by PLB with W. R. Binns, A. Chieffi, R. Engel, H. Kang, and D. Ryu are gratefully acknowledged. PLB also thanks Carola Dobrigkeit Chinellato and Roger Clay for helpful comments on the manuscript.

The article processing charges for this open-access publication were covered by the Max Planck Society.

Edited by: P. Desiati

Reviewed by: two anonymous referees

\section{References}

Aab, A., Abreu, P., Aglietta, M., et al. (Pierre Auger Collaboration): Depth of Maximum of Air-Shower Profiles at the Auger Observatory. I. Measurements at Energies above $10^{17.8} \mathrm{eV}$, Phys. Rev. D, 90, 122005, doi:10.1103/PhysRevD.90.122005, 2014a.

Aab, A., Abreu, P., Aglietta, M., et al. (Pierre Auger Collaboration): Depths of Maximum of Air-Shower Profiles at the Pierre Auger Observatory. II. Composition Implications, Phys. Rev. D, 90, 122006, doi:10.1103/PhysRevD.90.122006, 2014b.

Aab, A., Abreu, P., Aglietta, M., et al. (Pierre Auger Collaboration): Large scale distribution of ultra high energy cosmic rays detected at the Pierre Auger Observatory with zenith angles up to $80^{\circ}$, Astrophys. J., 802, 111 pp., 2015.

Aartsen, M. G., Ackermann, M., Adams, J., et al. (IceCube Collaboration): Observation of High-Energy Astrophysical Neutrinos in Three Years of IceCube Data, Phys. Rev. Lett., 113, 101101, doi:10.1103/PhysRevLett.113.101101, 2014.

Abbasi, R., Abdou, Y., Abu-Zayyad, T., et al. (IceCube Collaboration): An absence of neutrinos associated with cosmic-ray acceleration in gamma-ray bursts, Nature, 484, 351-354, 2012.

Abbasi, R., Bellido, J., Belz, J., et al. (Pierre Auger Collaboration, Telescope Array Collaboration): Report of the Working Group on the Composition of Ultra High Energy Cosmic Rays, eprint, arXiv:1503.07540, 2015.

Abbasi, R. U., Abe, M., Abu-Zayyad, T., et al. (Telescope Array Collaboration): Indications of Intermediate-scale Anisotropy of Cosmic Rays with Energy Greater Than $57 \mathrm{EeV}$ in the Northern Sky Measured with the Surface Detector of the Telescope Array Experiment, Astrophys. J. Lett., 790, L21, doi:10.1088/20418205/790/2/L21, 2014.

Aguilar, M., Aisa, D., Alvino, A., et al. (AMS Collaboration): Electron and Positron Fluxes in Primary Cosmic Rays Measured with the Alpha Magnetic Spectrometer on the International Space Station, Phys. Rev. Lett., 113, 121102, doi:10.1103/PhysRevLett.113.121102, 2014.

Ahn, H. S., Allison, P., Bagliesiet, M. G., et al. (CREAM Collaboration): Energy Spectra of Cosmic-ray Nuclei at High Energies, Astrophys. J., 707, 593-603, 2009.

Armstrong, J. W., Rickett, B. J., and Spangler, S. R.: Electron density power spectrum in the local interstellar medium, Astrophys. J., 443, 209-221, 1995.

Beck, R. and Hoernes, P.: Magnetic spiral arms in the galaxy NGC6946, Nature, 379, 47-49, 1996.

Beuermann, K., Kanbach, G., and Berkhuijsen, E. M.: Radio structure of the Galaxy - Thick disk and thin disk at $408 \mathrm{MHz}$, Astron. Astrophys., 153, 17-34, 1985.

Biermann, P. L.: Cosmic rays. 1. The cosmic ray spectrum between $10^{\prime} \mathrm{GeV}$ and 3 10' GeV, Astron. Astrophys., 271, 649-661, 1993.

Biermann, P. L.: Cosmic ray interactions in the Galaxy: Nuclear Astrophysics at Hirschegg, in Proc., GSI, Darmstadt, 211-222, 1998.

Biermann, P. L. and Strittmatter, P. A.: Synchrotron emission from shock waves in active galactic nuclei, Astrophys. J., 322, 643649, 1987.

Biermann, P. L., Langer, N., Eun-Suk Seo, and Stanev, T.: Cosmic Rays IX. Interactions and transport of cosmic rays in the Galaxy, Astron. Astrophys, 369, 269-277, 2001. 
Biermann, P. L., Becker, J. K., Meli, A., Rhode, W., Seo, E. S., and Stanev, T.: Cosmic Ray Electrons and Positrons from Supernova Explosions of Massive Stars, Phys. Rev. Lett., 103, 061101, doi:10.1103/PhysRevLett.103.061101, 2009.

Biermann, P. L., Becker, J. K., Caceres, G., Meli, A., Seo, E.-S., and Stanev, T.: The WMAP Haze from the Galactic Center Region Due to Massive Star Explosions and a Reduced Cosmic Ray Scale Height, Astrophys. J. Lett., 710, L53-L57, 2010.

Biermann, P. L., Becker Tjus, J., Seo, E.-S., and Mandelartz, M.: Cosmic-Ray Transport and Anisotropies, Astrophys. J., 768, 8 pp., doi:10.1088/0004-637X/768/2/124, 2013.

Binns, W. R.: Cosmic-Ray Origins, Science, 334, 1071-1072, 2011. Binns, W. R., Wiedenbeck, M. E. Arnould, M., Cummings, A. C., de Nolfo, G. A., Goriely, S., Israel, M. H., Leske, R. A., Mewaldt, R. A., Stone, E. C., and von Rosenvinge, T. T.: The OB association origin of galactic cosmic rays, New Astron. Rev., 52, 427430, 2008.

Binns, W. R., Christian, E. R., Cummings, A. C., De Nolfo, G. A., Israel, M. H., Leske, R. A., Mewaldt, R. A., Stone, E. C., Von Rosenvinge, T. T., and Wiedenbeck, M. E.: Constraints on Galactic Cosmic-Ray Origins from Elemental and Isotopic Composition Measurements, International Cosmic Ray Conference, 33rd, 2-9 July 2013, Rio de Janeiro, Brazil, 0646, 4 pp., 2013.

Brunetti, M. T. and Codino, A.: Age of Cosmic-Ray Protons Computed Using Simple Configurations of the Galactic Magnetic Field, Astrophys. J., 528, 789-798, 2000.

Chyźy, K. T., Soida, M., Bomans, D. J., Vollmer, B., Balkowski, Ch., Beck, R., and Urbanik, M.: Large-scale magnetized outflows from the Virgo Cluster spiral NGC 4569. A galactic wind in a ram pressure wind, Astron. Astrophys., 447, 465-472, 2006.

Chyźy, K. T., Bomans, D. J., Krause, M., Beck, R., Soida, M., and Urbanik, M.: Magnetic fields and ionized gas in nearby late type galaxies, Astron. Astrophys., 462, 933-941, 2007.

Compton, A. H. and Getting, I. A.: An Apparent Effect of Galactic Rotation on the Intensity of Cosmic Rays, Phys. Rev. Lett., 47, 817, doi:10.1103/PhysRev.47.817, 1935.

Cox, D. P.: Cooling and Evolution of a Supernova Remnant, Astrophys. J., 178, 159-168, 1972.

Cox, D. P. and Smith, B. W.: Large-Scale Effects of Supernova Remnants on the Galaxy: Generation and Maintenance of a Hot Network of Tunnels, Astrophys. J. Lett., 189, L105-L108, 1974.

Das, S., Kang, H., Ryu, D., and Cho, J.: Propagation of Ultra-HighEnergy Protons through the Magnetized Cosmic Web, Astrophys. J., 682, 29-38, 2008.

Drury, L. O'C: An introduction to the theory of diffusive shock acceleration of energetic particles in tenuous plasmas, Rep. Prog. Phys., 46, 973-1027, 1983.

Erice CR meeting: Erice July 2014 lectures, available at: http://laspace.lsu.edu/ISCRA/ISCRA2014/Presentations.php, last acess: September 2015, 2014.

Everett, J., Zweibel, E. G., Benjamin, R. A., McCammon, D., Rocks, L., and Gallagher III, J. S.: The Milky Way's KiloparsecScale Wind: A Hybrid Cosmic-Ray and Thermally Driven Outflow, Astrophys. J., 674, 258-270, 2008.

Everett, J., Zweibel, E., Benjamin, B., McCammon, D., Schiller, Q., Rocks, L., and Gallagher III, J. S.: Cosmic Ray- and ThermalPressure Driven Winds: Does the Milky Way Host a Kpc-Scale Outflow?, in: The Role of the Disk-Halo Interaction in Galaxy
Evolution: Outflow vs. Infall?, edited by: de Avillez, M. A., EAS Publications Series, 56, 73-76, 2012.

Fraternale, F., Gallana, L., Iovieno, M., Opher, M., Richardson, J. D., and Tordella, D.: Turbulence in the solar wind: spectra from Voyager 2 data at $5 \mathrm{AU}$, eprint, arXiv:1502.07114, 2015.

Gaisser, T. K., Stanev, T., and Tilav, S.: Cosmic ray energy spectrum from measurements of air showers, Frontiers of Physics, 8, 748758, 2013.

Gizani, N. A. B. and Leahy, J. P.: A multiband study of Hercules A - II. Multifrequency VLA imaging, Mon. Not. R. Astron. Soc., 342, 399-421, 2003.

Goldstein, M. L., Roberts, D. A., and Matthaeus, W. H.: Magnetohydrodynamic Turbulence In The Solar Wind, Annu. Rev. Astron. Astr., 33, 283-326, 1995.

Gopal-Krishna, Biermann, P. L., de Souza, V., and Wiita, P. J.: Ultra-high-energy Cosmic Rays from Centaurus A: Jet Interaction with Gaseous Shells, Astrophys. J. Lett., 720, L155-L158, 2010.

Gregorini, L., Mantovani, F., Eckart, A., Biermann, P., Witzel, A., and Kühr, H.: The low-frequency spectra of a complete sample of extragalactic radio sources, Astron. J., 89, 323-331, 1984.

Haverkorn, M. and Spangler, S. R.: Plasma Diagnostics of the Interstellar Medium with Radio Astronomy, Space Sci. Rev., 178, 483-511, 2013.

Iacobelli, M., Haverkorn, M., Orrú, E., et al.: Studying Galactic interstellar turbulence through fluctuations in synchrotron emission. First LOFAR Galactic foreground detection, Astron. Astrophys., 558, A72, doi:10.1051/0004-6361/201322013, 2013.

Iacobelli, M., Burkhart, B., Haverkorn, M., Lazarian, A., Carretti, E., Staveley-Smith, L., Gaensler, B. M., Bernardi, G., Kesteven, M. J., and Poppi, S.: Galactic interstellar turbulence across the southern sky seen through spatial gradients of the polarization vector, Astron. Astrophys., 566, 12 pp., 2014.

Kardashev, N. S.: Nonstationarity of Spectra of Young Sources of Nonthermal Radio Emission, Astron. Zh., 39, 393-409, 1962; translation: Sov. Astr. A. J., 6, 317-327, 1962.

Kolmogorov, A.: The Local Structure of Turbulence in Incompressible Viscous Fluid for Very Large Reynolds' Numbers, Dokl. Akad. Nauk SSSR, 30, 301-305, 1941a.

Kolmogorov, A.: Dissipation of Energy in Locally Isotropic Turbulence, Dokl. Akad. Nauk SSSR, 32, p. 16, 1941 b.

Kotera, K. and Olinto, A. V.: The Astrophysics of Ultrahigh-Energy Cosmic Rays, Annu. Rev. Astron. Astr., 49, 119-153, 2011.

Lagage, P. O. and Cesarsky, C .J.. The maximum energy of cosmic rays accelerated by supernova shocks, Astron. Astrophys., 125, 249-257, 1983.

Lemoine, M., Sigl, G., Olinto, A. V., and Schramm, D. N.: Ultrahigh-Energy Cosmic-Ray Sources and Large-Scale Magnetic Fields, Astrophys. J. Lett., 486, L115-L118, 1997.

Letessier-Selvon, A.: Highlights from the Pierre Auger Observatory, Braz. J. Phys., 44, 560-570, 2014.

Lovelace, R. V. E.: Dynamo model of double radio sources, Nature, 262, 649-652, 1976.

Meli, A., Becker, J. K., and Quenby, J. J.: On the origin of ultra high energy cosmic rays: subluminal and superluminal relativistic shocks, Astron. Astrophys., 492, 323-336, 2008.

Mirabel, I. F.: Microquasars: Summary and Outlook, in: The Jet Paradigm, Lecture Notes in Physics, Springer-Verlag Berlin Heidelberg, 794, 1-15, 2010. 
Parker, E.: Dynamics of the Interplanetary Gas and Magnetic Fields, Astrophys. J., 128, 664-676, 1958.

Ptuskin, V. S., Lukasiak, A., Jones, F. C., and Webber, W. R.: The Modified Weighted Slab Technique: Results, edited by: Kieda, D., Salamon, M., and Dingus, B., Proceedings of the 26th International Cosmic Ray Conference, 17-25 August 1999, Salt Lake City, Utah, USA, 4, p. 291, 1999.

Rossa, J. and Dettmar, R.-J.: An Halpha survey aiming at the detection of extraplanar diffuse ionized gas in halos of edge-on spiral galaxies. I. How common are gaseous halos among non-starburst galaxies?, Astron. Astrophys., 406, 493-503, 2003.

Sarkar, K. C., Nath, B. B., Sharma, P., and Shchekinov, Y.: Long way to go: how outflows from large galaxies propagate through the hot halo gas, Mon. Not. R. Astron. Soc., 448, 328-343, 2015.

Smith, L. F., Biermann, P., and Mezger, P. G.: Star formation rates in the Galaxy, Astron. Astrophys., 66, 65-76, 1978.

Snowden, S. L., Egger, R., Freyberg, M. J., McCammon, D., Plucinsky, P. P., Sanders, W. T., Schmitt, J. H. M. M., Trümper, J., and Voges, W.: ROSAT Survey Diffuse X-Ray Background Maps. II., Astrophys. J., 485, 125-135, 1997.
Todero Peixoto, C. J., de Souza, V., and Biermann, P. L.: Cosmic rays: the spectrum and chemical composition from $10^{10}$ to $10^{20} \mathrm{eV}$, J. Cosmol. Astropart. P., 07, 042, doi:10.1088/14757516/2015/07/042, 2015.

Tüllmann, R., Breitschwerdt, D., Rossa, J., Pietsch, W., and Dettmar, R.-J.: The multi-phase gaseous halos of star-forming late-type galaxies - II. Statistical analysis of key parameters, Astron. Astrophys., 457, 779-785, 2006.

Uhlig, M., Pfrommer, C., Sharma, M., Nath, B. B., Enßlin, T. A., and Springel, V.: Galactic winds driven by cosmic ray streaming, Mon. Not. R. Astron. Soc., 423, 2374-2396, 2012.

Witzel, A., Schalinski, C. J., Johnston, K. J., Biermann, P. L., Krichbaum, T. P., and Hummel, C. A.: The occurrence of bulk relativistic motion in compact radio sources, Astron. Astrophys., 206, 245-252, 1988.

Yan, H. and Lazarian, A.: Cosmic Ray Transport in MHD Turbulence: Large and Small Scale Interactions, in: Numerical modeling of space plasma flows, edited by: Pogorelov, N. V., Font, J. A., Audit, E., and Zank, G. P., Astronomical Society of the Pacific Conference Series, 459, p. 40, 2012. 